\title{
Squeezing of open boundaries by Maxwell-consistent real coordinate transformation
}

\author{
Shyroki, Dzmitry
}

Published in:

IEEE Microwave and Wireless Components Letters

Link to article, DOI:

10.1109/LMWC.2006.884768

Publication date:

2006

Document Version

Publisher's PDF, also known as Version of record

Link back to DTU Orbit

Citation (APA):

Shyroki, D. (2006). Squeezing of open boundaries by Maxwell-consistent real coordinate transformation. IEEE Microwave and Wireless Components Letters, 31(11), 576-578. https://doi.org/10.1109/LMWC.2006.884768

\section{General rights}

Copyright and moral rights for the publications made accessible in the public portal are retained by the authors and/or other copyright owners and it is a condition of accessing publications that users recognise and abide by the legal requirements associated with these rights.

- Users may download and print one copy of any publication from the public portal for the purpose of private study or research.

- You may not further distribute the material or use it for any profit-making activity or commercial gain

- You may freely distribute the URL identifying the publication in the public portal

If you believe that this document breaches copyright please contact us providing details, and we will remove access to the work immediately and investigate your claim. 


\title{
Squeezing of Open Boundaries by Maxwell- Consistent Real Coordinate Transformation
}

\author{
Dzmitry M. Shyroki
}

\begin{abstract}
To emulate open boundaries within a finite computational domain, real-function coordinate transformation consistent with generally covariant Maxwell equations is proposed. The mapping-realized with arctangent function here-has a transparent geometric meaning of pure squeezing of coordinates, does not introduce artificially lossy layers (or "lossy coordinates") to absorb outgoing radiation, nor lead to spurious non-Maxwellian fields. In finite-difference frequency-domain calculations on staggered grid, clear superiority over perfectly matched layers is demonstrated by the proposed technique, at a lower computation cost, in drastic elimination of parasitic coupling of guided modes to the boundaries of the computational window.
\end{abstract}

Index Terms-Absorbing boundary conditions (ABCs), coordinate transformation, Maxwell equations, perfectly matched layer (PML).

\section{INTRODUCTION}

D IRECT numerical methods of modeling electromagnetic phenomena, such as finite-difference time-domain (FDTD) and frequency-domain (FDFD) schemes, are invariably concerned with how to represent infinite space surrounding the region of interest on a bounded computation domain. During the last ten years, a dominating technique to emulate open boundaries was by means of absorbing perfectly matched layers (PMLs) introduced originally in [1]. Three major advantages of PMLs are: 1) locality, enabling straightforward parallelization in FDTD and use of sparse matrices in FDFD; 2) efficiency in wide-angle, wide-band suppressing of reflectivity with a fairly small number of PM sublayers; and 3) ease of integration into existing finite-difference Maxwell solvers-especially in non-split, or anisotropic PML formulation [2].

There is one conceptually dissatisfactory issue with PMLs, however. From their inception, PMLs were designed to emulate open, i.e., infinitely distant boundaries with absorbing boundary conditions (ABCs) exhibiting, ideally, zero reflectivity. However satisfactory for transient scattering simulations such mimicry might be, it is not readily justified in guided-and cavity-mode analysis, be it in frequency or in time domain, insofar as confined modes having their tails extending outside the region of confinement are inevitably perturbed by any modification of electromagnetic properties of outer space. With this motivation to improve open boundary conditions for confined-mode analysis while preserving the renowned merits of standard PMLs, we propose in this letter a simple

Manuscript received April 4, 2006; revised July 3, 2006.

The author is with the Department of Communications, Optics and Materials, Technical University of Denmark, Lyngby 2800, Denmark (e-mail: ds@com. dtu.dk).

Digital Object Identifier 10.1109/LMWC.2006.884768 and straightforward method of mapping infinite surrounding space onto the finite computational domain, instead of inserting anisotropic absorbing PMLs between the region of interest and computation boundaries.

We do the mapping by transforming dielectric permittivity $\epsilon$ and magnetic permeability $\mu$ while retaining the form of Maxwell equations untouched, exploiting the general covariance of electrodynamics - an appropriate formulation is given in Section II. Such mapping-illustrated by use of arctangent function here, introduced in Section III-is rigorous at the stage of analytic description; is local, retaining locality of basic finite-difference schemes; is real-valued, enabling finite-difference algorithms in real notation where appropriate; and is remarkably effective compared to the PML technique, as demonstrated by FDFD tests presented in Section IV. This method, however, is not aimed to rule out conventional PMLs, but rather to occupy a niche-guided- and cavity-mode analysis, in the first place-for which PMLs are intrinsically not so good; and to assist PMLs whenever impedance-matched absorbers are to be used-for example, to obtain complex propagation constants for leaky modes in frequency domain, or to damp scattered field near domain boundaries in time domain simulations.

\section{Covariant MaXwell EQuations}

It was Lorentz-covariance of Maxwell equations that led to special relativity over a century ago. Another, less celebrated though well and long ago established fact about Maxwell equations is that they can be formulated in a generally covariant manner, so that they do not change their form under arbitrary reversible transformation from Cartesian coordinates [3]. Surprisingly, this feature was first exploited in direct computational electromagnetics perhaps only a decade ago, in "logically Cartesian" FDTD simulations of high index contrast dielectric structures on non-Cartesian grids [4], [5]. Starting from covariant formulation of [3], let us write Maxwell equations here in terms of an electric covariant vector $E_{\nu}$ and a magnetic covariant pseudovector $\tilde{H}_{\nu}$

$$
\begin{aligned}
& \tilde{\mathfrak{E}}^{\kappa \lambda \nu} \partial_{\lambda} E_{\nu}=-\mu^{\kappa \lambda} \dot{\tilde{H}}_{\lambda}, \quad \partial_{\kappa} \mu^{\kappa \lambda} \tilde{H}_{\lambda}=0 \\
& \tilde{\mathfrak{E}}^{\kappa \lambda \nu} \partial_{\lambda} \tilde{H}_{\nu}=\epsilon^{\kappa \lambda} \dot{E}_{\lambda}+\mathfrak{j}^{\kappa}, \quad \partial_{\kappa} \epsilon^{\kappa \lambda} E_{\lambda}=\rho
\end{aligned}
$$

a form which, written in components explicitly, is identical to conventional Cartesian representation (pseudo permutation field $\tilde{\mathfrak{E}}^{\kappa \lambda \nu}$ equals Levi-Civita symbol in any coordinate system; $\kappa$, $\lambda, \mu=1, \ldots, 3)$, with the constitutive relations

$$
\tilde{\mathfrak{B}}^{\lambda}=\mu^{\lambda \nu} \tilde{H}_{\nu}, \quad \mathfrak{D}^{\lambda}=\epsilon^{\lambda \nu} E_{\nu}
$$

(i.e., no optical activity assumed), where $\tilde{\mathfrak{B}}^{\lambda}$ is magnetic induction pseudo vector density of weight +1 , and $\mathfrak{D}^{\lambda}$ is electric 
induction vector density; hence $\epsilon^{\lambda \nu}$ and $\mu^{\lambda \nu}$ are contravariant tensor densities transformed according to

$$
\epsilon^{\lambda^{\prime} \nu^{\prime}}=|\Delta|^{-1} J_{\lambda}^{\lambda^{\prime}} J_{\nu}^{\nu^{\prime}} \epsilon^{\lambda \nu}, \quad \mu^{\lambda^{\prime} \nu^{\prime}}=|\Delta|^{-1} J_{\lambda}^{\lambda^{\prime}} J_{\nu}^{\nu^{\prime}} \mu^{\lambda \nu}
$$

where $J_{\lambda}^{\lambda^{\prime}} \equiv \partial_{\lambda} x^{\lambda^{\prime}}$ is the Jacobian transformation matrix for contravariant components, $\Delta \equiv \operatorname{det} J_{\lambda}^{\lambda^{\prime}}$ is its determinant.

Such formulation enables one to hide all metric information into $\epsilon^{\lambda \nu}$ and $\mu^{\lambda \nu}$ while invariably using Cartesian-like representation of Maxwell equations (1) and (2), which is extremely convenient; no wonder many authors strived (successfully) to "derive" (4) on different grounds and under different assumptions, as in [4] for isotropic media or in [6] for diagonal transformation matrices. It is worth noting that, however, (4) is a direct consequence of transformation characteristics assigned to the electric and magnetic fields in covariant electromagnetics, so that no tricky derivations are needed. In practice, dielectric permittivity and magnetic permeability are referenced to a Cartesian frame, so if one wants to use non-Cartesian coordinates instead, with Cartesian-like equations (1) and (2), then transformation rules (4) are to be employed and specified for transformation from Cartesian to those coordinates. We make such specification for the mapping onto arctangent-squeezed coordinates in the next section.

\section{OPEN BOUNDARIES ON CONFINED DOMAIN}

Let the Cartesian coordinates $x, y, z$ (unprimed) be transformed to $x^{\prime}, y^{\prime}, z^{\prime}$ (primed) with

$$
\left\{\begin{array}{l}
x^{\prime}=\arctan \left(x / x_{0}\right) \\
y^{\prime}=\arctan \left(y / y_{0}\right) \\
z^{\prime}=\arctan \left(z / z_{0}\right)
\end{array}\right.
$$

where $x_{0}, y_{0}$, and $z_{0}$ are characteristic lengths along the corresponding coordinates. (Though arbitrary at analytic stage, for adequate sampling on squeezed grid these lengths should be adjusted to actual dimensions of the region of interest or to the wavelength.) By differentiating (5) one gets

$$
J_{\lambda}^{\lambda^{\prime}}=\left(\begin{array}{ccc}
x_{0}^{-1} \cos ^{2} x^{\prime} & 0 & 0 \\
0 & y_{0}^{-1} \cos ^{2} y^{\prime} & 0 \\
0 & 0 & z_{0}^{-1} \cos ^{2} z^{\prime}
\end{array}\right) .
$$

The "squeezed" permittivity and permeability can be obtained with (4) and (6) immediately; one may write

$$
\epsilon^{x^{\prime} x^{\prime}}\left(x^{\prime}, y^{\prime}, z^{\prime}\right)=\epsilon^{x x}\left(x^{\prime}, y^{\prime}, z^{\prime}\right) \cdot J_{x}^{x^{\prime}} /\left(J_{y}^{y^{\prime}} J_{z}^{z^{\prime}}\right)
$$

and similarly for other components of $\epsilon^{\prime}$ and $\mu^{\prime}$, to retain similarity to anisotropic PMLs with "stretching variables" [7] $s_{x}^{-1}=$ $J_{x}^{x^{\prime}}$ etc. It should be emphasized though that transformation (5) employs real-valued functions and bears a clear meaning of pure squeezing of space, unlike "complex coordinate stretching" [7] or "lossy mapping of space" [8] used to derive standard (lossy) PMLs by adding complex-valued "degrees of freedom" to spatial variables in Maxwell equations. Note also that even if matrix representations of dielectric permittivity and magnetic permeability are non-diagonal in an initial (unprimed) coordinate system, this generates no additional difficulty in deriving $\epsilon^{\prime}$ and $\mu^{\prime}$; the squeezing of cylindrical or spherical coordinates, or any other non-Cartesian or non-orthogonal coordinates if preferred, also poses no difficulty in the present approach.
Transformation (5) is smooth; and the price for the smoothness is that it is linear only in the infinitesimal vicinity of the origin of coordinates, so that familiar geometric figures become distorted on new mesh and, analytically, are defined by equations with $x, y$, and $z$ coordinates replaced by $x_{0} \tan x^{\prime}$ etc. This may produce some overhead when generating $\epsilon$ and $\mu$ profiles on a squeezed grid and poses high demands on index averaging at index discontinuities for better numerical convergence. To go around that, one might introduce piecewise mapping functions like $x^{\prime}=x$ for $|x| \leq a, x^{\prime}=a+b \frac{2}{\pi} \arctan \frac{\pi}{2} \frac{x-a}{b}$ for $|x|>a$, ending up with $b$-wide "squeeze-transform layers" (STLs) inside a $2(a+b)$-wide computation domain. Next we compare performance of STLs against PMLs of similar width in frequency-domain simulations.

\section{FDFD SimUlations OF GUIDED MODES}

In this section, a fundamental mode in step-index fiber is considered for which analytic solution is known [9]. The fiber is characterized by core-to-cladding index contrast 1.515 to 1.5 as in [10], core radius $R$, and is centered in the $3 R \times 3 R$ computational window. This fiber is single-moded for wavelengths $\lambda / R>0.556$, corresponding to normalized frequencies $v<2.405$. Full-vector FDFD algorithm on staggered equidistant grid is used, adapted to the two-dimensional (2-D) geometry; discretization is performed at the first-order Maxwell equations level, in particular because this provides better PML performance than when a second-order Helmholtz equation is discretized [11]. For PMLs, we chose a quadratic conductivity profile, as $\sigma_{x}=\sigma_{\max }(x-a)^{2} / b^{2},|x|>a$, and put $\sigma_{\max }=10$ to produce fairly stable results over the whole range of wavelengths considered.

The accuracy of guided-mode calculations with zero boundary conditions, without any special treatment, is admissible at short wavelengths but rapidly deteriorates with increasing $\lambda / R$, as seen in Fig. 1. Transforming $\epsilon$ and $\mu$ in narrow regions inside the domain boundaries as prescribed by PML formulation delays the increase of errors with wavelength, but the results are still imperfect, in terms of both accuracy and sensitivity to the layer width (we included $1 / 10$-wide and $1 / 20$-wide layers, as related to the whole domain, which gives eight and four sublayers for the chosen resolution, correspondingly). Memory storage requirements and calculation time also rise since system matrix inevitably becomes complex-valued with PMLs. Using STLs instead is a real remedy: with the same computation effort as that of pure zero-boundary calculations, accuracy improves dramatically over a wide wavelength range.

Convergence for the fundamental mode index at a cut-off wavelength $\lambda / R=0.556$ with increasing resolution is shown in Fig. 2, for zero boundaries and, again, for $1 / 10$-wide and $1 / 20$-wide PMLs and STLs. It is remarkable how accurate and rapidly converging the results of STL-assisted calculations are. Note also that such performance is achieved with fairly narrow layers; this alleviates the problem of finding an optimal mapping function for STLs, as opposed to the problem of optimal conductivity profile of PMLs. Fig. 3 further supports the suggestion that performance of STLs is not restrained tightly to a particular region in the parameter space of $a, b$, and mapping function profile, since a simple "brute-force" increase in resolution, as demonstrated for the arbitrarily chosen $1 / 10$-wide arc- 


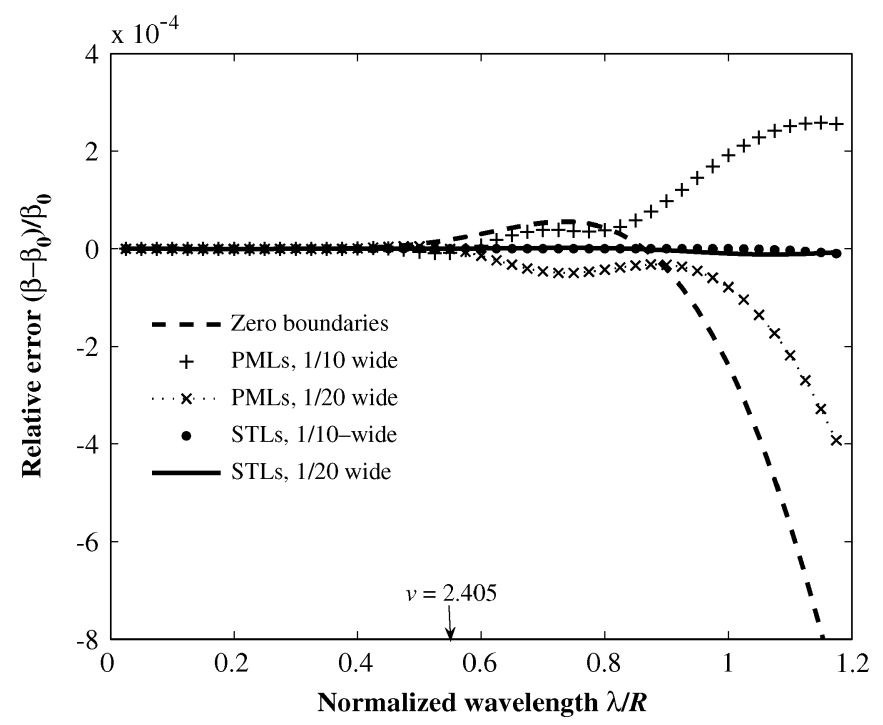

Fig. 1. Accuracy of fundamental mode dispersion calculations with different boundary conditions. Computation domain is discretized in $80 \times 80$ grid cells; the widths of the layers are $1 / 10$ or $1 / 20$ of the whole domain width.

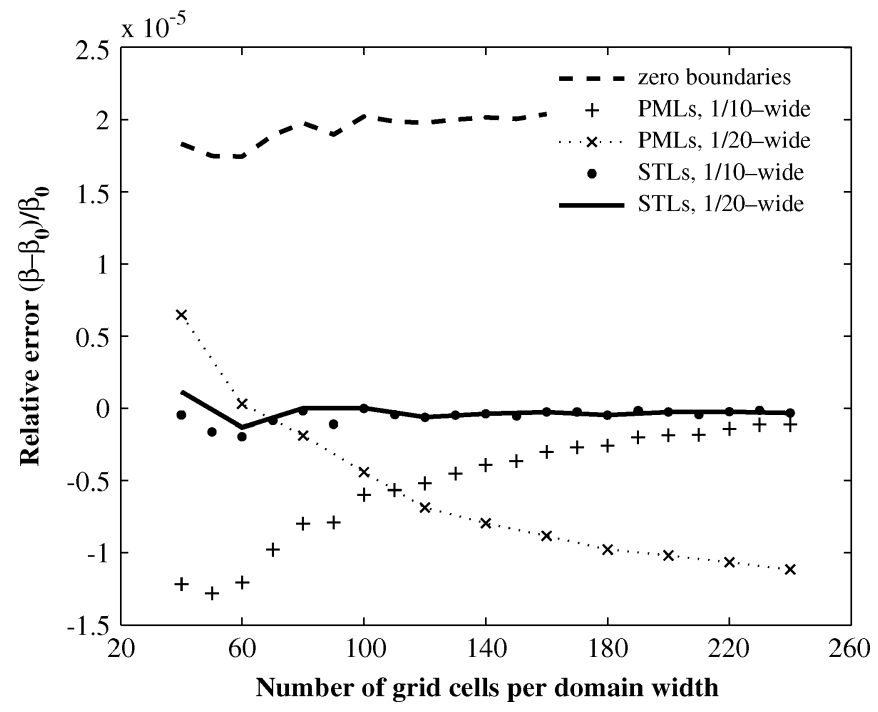

Fig. 2. Convergence of fundamental mode index at $\lambda / R=0.556$, compared for zero-boundary calculations and STL- and PML-assisted results. The widths of ST and PM regions are, again, 1/10 or 1/20 of the whole domain.

tangent-squeezed layer, improves the accuracy at longer wavelengths nearly linearly.

\section{CONCLUSION}

The squeezing of coordinates to emulate open boundaries in finite-difference computations is proposed. The employed nonlinear squeezing of $\epsilon$ and $\mu$ into a bounded domain, after equidistant discretization on that domain, results in better "physical" sampling for the central region of space and progressively coarser for outskirts, and thus can be regarded as a kind of adaptive meshing technique. At the methodological level, this approach is attractive due to its conceptual clarity: we do not surround the computation window with artificial lossy media;

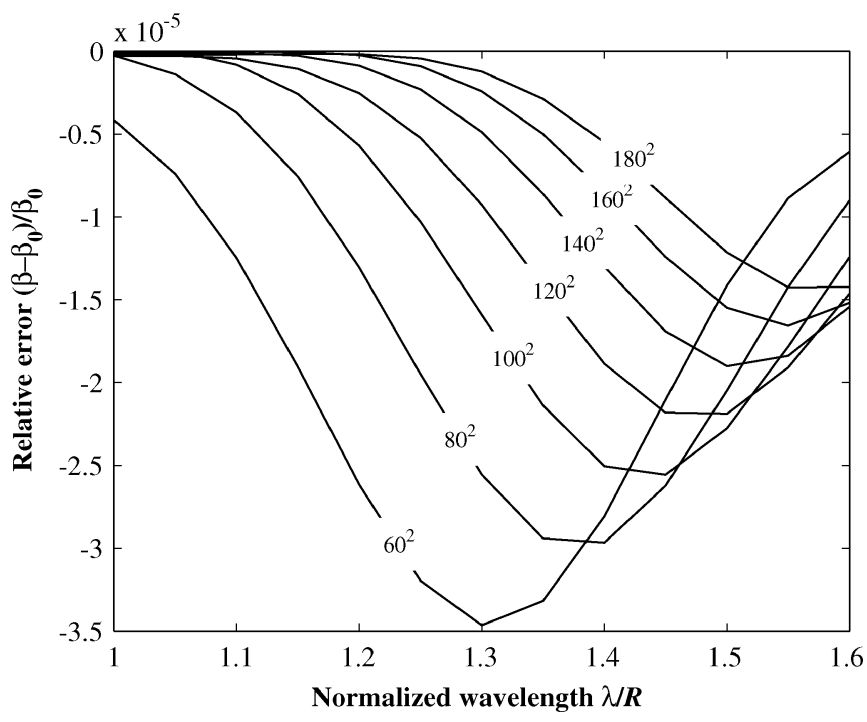

Fig. 3. Accuracy of fundamental mode dispersion calculations employing 1/10-wide STLs, at longer wavelengths than in Fig. 1, with resolutions ranging from $60 \times 60$ to $180 \times 180$ grid cells over the whole domain.

we do not modify the Maxwell equations in any way; all we do is we choose a coordinate system allowed by the covariant nature of Maxwell equations and is suitable for calculations. While in the time domain the method is yet to be applied, the frequency-domain simulations of guided propagation presented here demonstrate excellent performance of squeeze-transform layers (STLs) compared to PMLs. And whenever impedancematched absorbers at the boundaries should be included, traditional PMLs can be used in combination with real-function coordinate squeezing.

\section{REFERENCES}

[1] J. P. Bérenger, "A perfectly matched layer for the absorption of electromagnetic waves," J. Comput. Phys., vol. 114, pp. 185-200, 1994.

[2] S. Gedney, "An anisotropic perfectly matched layer-absorbing medium for the truncation of FDTD lattices," IEEE Trans. Antennas Propag., vol. 44, no. 12, pp. 1630-1639, Dec. 1996.

[3] E. J. Post, Formal Structure of Electromagnetics. Amsterdam, The Netherlands: North-Holland, 1962.

[4] A. J. Ward and J. B. Pendry, "Refraction and geometry in Maxwell's equations," J. Modern Opt., vol. 43, pp. 773-793, 1996.

[5] _ "Calculating photonic Green's functions using a nonorthogonal finite-difference time-domain method," Phys. Rev. B, vol. 58, pp. $7252-7259,1998$.

[6] F. L. Teixeira and W. C. Chew, "General closed-form PML constitutive tensors to match arbitrary bianisotropic and dispersive linear media," IEEE Microw. Guided Wave Lett., vol. 8, no. 5, pp. 223-225, May 1998.

[7] W. C. Chew and W. H. Weedon, "A 3D perfectly matched medium from modified Maxwell's equations with stretched coordinates," Microw. Opt. Technol. Lett., vol. 7, pp. 599-604, 1994.

[8] C. M. Rappaport, "Perfectly mathed absorbing boundary conditions based on anisotropic lossy mapping of space," IEEE Microw. Guided Wave Lett., vol. 5, no. 3, pp. 90-92, Mar. 1995.

[9] D. Marcuse, Theory of Dielectric Optical Waveguides, 2nd ed. San Diego, CA: Academic, 1991.

[10] Y. Tsuji and M. Koshiba, "Guided-mode and leaky-mode analysis by imaginary distance beam propagation method based on finite element scheme," J. Lightw. Technol., vol. 18, pp. 618-623, 2000.

[11] C. M. Rappaport, M. Kilmer, and E. Miller, "Accuracy considerations in using the PML ABC with FDFD Helmholtz equation computation," Int. J. Numer. Model., vol. 13, pp. 471-482, 2000. 